Editorial

\title{
Preparation for ISAPA 2021 in the midst of COVID-19
}

\author{
Pauli Rintala ${ }^{1}$ \\ Received: $12^{\text {th }}$ October; Accepted: $16^{\text {th }}$ November 2020; Published: $17^{\text {th }}$ November 2020
}

COVID-19 has had an enormous effect on the whole world; physically, mentally, socially and economically. Planning and hosting international gatherings and scientific symposiums such as the International Symposium of Adapted Physical Activity (ISAPA) need reevaluation in this new world situation. Many events have been cancelled or postponed. The pandemic has given everyone a taste of COVID-19 one way or another. It is obvious that the negative effects are vastly greater than the positive ones. In the competitive sports world, we do not even know what the outcomes will be considering the continuation of competitions such as Paralympics. Will the sports clubs survive over the pandemic? Moreover, will the wellbeing of citizens decrease due to the lack of physical activity opportunities? People with disabilities are vulnerable in this pandemic. There is the risk that community efforts to deliver physical activity programs may focus less on physical activity and wellbeing of people with disability in the future (Fitzgerald, Stride, \& Drury, 2020).

At the individual level, the pandemic has forced people to alter their daily routines (Lim \& Pranata, 2020). Some exercisers have taken up more outdoor opportunities (Mutz \& Gerke, 2020) where keeping to safe distance rules are easier to adhere to than to being indoors. Advise for outdoor activities appeared in public health messages. There are more and more people who live in cities leading to fewer outdoor activity possibilities. At the same time, there have been many online innovations to adapt physical activity $(\mathrm{Ng}, 2020)$ that can be performed in small spaces, and even indoors.

The nature of Adapted Physical Activity is to be creative and innovative. Professionals, such as health promotion specialists or physical education teachers do not always know how to specifically apply sports, physical activity, or exercise for individuals with various disabilities. In those circumstances, professionals have to find a solution or adaptation, whether it is a modification of equipment, or a means to get someone to the sport venue. I have learned in the early years of my career that one of the most important attributes as an Adapted Physical Activity specialist is to possess creativity. Having the right attitude, knowledge and skills are crucial when working with individuals who have disabilities but being creative is needed in new situations (Sherrill, 2004). This is a lesson I learnt from Professor Claudine Sherrill, Texas Woman's University, in early 90's. Now with the COVID19 pandemic, we are practically in a new situation, in which creativity and innovations are valuable.

Collaborations between professionals must continue in spite of the pandemic $(\mathrm{Ng}$, 2020). An innovative solution emerged when the European Conference of Adapted Physical Activity was transformed into a one-day online seminar earlier this Autumn. Moreover, the North American Conference was cancelled, and replaced by several Adapted Physical Activity -webinars. Such events have opened up the opportunities for online collaborations in the coming years. The worry I have here in Finland, as the chair of the organizing committee for the next International Symposium of Adapted Physical Activity (ISAPA) scheduled for June 2021 (see: www.jyu.fi/isapa2021 ), is what to do. At this moment, the organizing committee has decided to deliver an online only conference after deciding against 
the hybrid model where face-to-face and remote attendees participate. As a result, Adapted Physical Activity professionals from around the world will miss an opportunity to visit Jyväskylä, Finland to engage with colleagues, share cultures, practices, research ideas, and collaborate creatively.

An online ISAPA would be organized for the first time in the ISAPA 40+ year history. On the positive side, the Online ISAPA may enable more people around the world to attend. Modern technology allows for streaming with other features for presenters and attendees at the symposium. Moreover, with this experience, ISAPA attendees may find virtual communication favorable in the future. The continued pandemic has already given potential attendees the significant experience in interacting via online sessions.

The theme of the 2021 ISAPA is "Quality partnerships in Adapted Physical Activity: Stronger together!" Well established partnerships are very essential, especially in the circumstances we live now due to the COVID-19 pandemic. Building strong, sustainable, and innovative partnerships is critical for the advancement of the interdisciplinary field of Adapted Physical Activity. Can ISAPA achieve the goals of this theme virtually in the context of the pandemic? The organizing committee is determined to address this issue creatively by the help of modern technology. In this way, online events provide new and extra opportunities to collaborate in the future, in addition to face-to-face contact.

\section{Author affiliations:}

1 Faculty of Sport and Health Sciences, University of Jyväskylä, Finland. Pauli.Rintala@jyu.fi

\section{References}

Fitzgerald, H., Stride, A., \& Drury, S. (2020). COVID-19, lockdown and (disability) sport. Managing Sport and Leisure. https://doi.org/10.1080/23750472.2020.1776950

Lim, M.A., \& Pranata, R. (2020). Sports activities during any pandemic lockdown. Irish Journal of Medical Science, (1971-). https://doi.org/10.1007/s11845-020-02300-9

Mutz, M., \& Gerke, M. (2020). Sport and exercise in times of self-quarantine: How Germans changed their behaviour at the beginning of the Covid-19 pandemic. International Review for the Sociology of Sport, 1-12. https://doi.org/10.1177/1012690220934335

$\mathrm{Ng}, \mathrm{K}$. (2020). Adapted physical activity through COVID-19. European Journal of Adapted Physical Activity, 13(1):1. https://doi.org/10.5507/euj.2020.003

Sherrill, C. (2004). Adapted Physical Activity, Recreation, and Sport: Cross-disciplinary and Lifespan. Dubuque, IA: McGraw Hill.

(C) 2020 by the authors. Submitted for possible open access publication under the terms and conditions of the Creative Commons Attribution (CC BY) license (http://creativecommons.org/licenses/by/4.o/). 